\title{
Genetic Influences on Cost-Efficient Organization of Human Cortical Functional Networks
}

\author{
Alex Fornito, ${ }^{1,2}$ Andrew Zalesky, ${ }^{2}$ Danielle S. Bassett, ${ }^{1,3}$ David Meunier, ${ }^{1}$ Ian Ellison-Wright, ${ }^{1,4}$ Murat Yücel, ${ }^{2}$ \\ Stephen J. Wood, ${ }^{2,5}$ Karen Shaw, ${ }^{2}$ Jennifer 0'Connor, ${ }^{2}$ Deborah Nertney, ${ }^{6}$ Bryan J. Mowry, ${ }^{6,7}$ Christos Pantelis, ${ }^{2}$ \\ and Edward T. Bullmore ${ }^{1,8}$ \\ ${ }^{1}$ Brain Mapping Unit, Behavioural \& Clinical Neurosciences Institute, University of Cambridge, Cambridge CB2 3EB, United Kingdom, ${ }^{2} \mathrm{Melbourne}$ \\ Neuropsychiatry Centre, Department of Psychiatry, University of Melbourne and Melbourne Health, Carlton South, Victoria 3053, Australia, ${ }^{3} \mathrm{Genes}$, \\ Cognition \& Psychosis Program, Clinical Brain Disorders Branch, National Institute of Mental Health, National Institutes of Health, Bethesda, Maryland \\ 20892, ${ }^{4}$ Avon and Wiltshire Mental Health Partnership NHS Trust, Salisbury SP2 7EP, United Kingdom, ${ }^{5}$ School of Psychology, University of Birmingham, \\ Edgbaston B15 2TT, United Kingdom, ${ }^{6}$ Queensland Centre for Mental Health Research,University of Queensland, The Park, Wacol, Queensland 4076, \\ Australia, ${ }^{7}$ Queensland Brain Institute, The University of Queensland, Brisbane, Queensland 4072, Australia, and ${ }^{8}$ GSK Clinical Unit Cambridge, \\ Addenbrooke's Hospital, Cambridge CB2 0QQ, United Kingdom
}

The human cerebral cortex is a complex network of functionally specialized regions interconnected by axonal fibers, but the organizational principles underlying cortical connectivity remain unknown. Here, we report evidence that one such principle for functional cortical networks involves finding a balance between maximizing communication efficiency and minimizing connection cost, referred to as optimization of network cost-efficiency. We measured spontaneous fluctuations of the blood oxygenation level-dependent signal using functional magnetic resonance imaging in healthy monozygotic (16 pairs) and dizygotic (13 pairs) twins and characterized cost-efficient properties of brain network functional connectivity between 1041 distinct cortical regions. At the global network level, $60 \%$ of the interindividual variance in cost-efficiency of cortical functional networks was attributable to additive genetic effects. Regionally, significant genetic effects were observed throughout the cortex in a largely bilateral pattern, including bilateral posterior cingulate and medial prefrontal cortices, dorsolateral prefrontal and superior parietal cortices, and lateral temporal and inferomedial occipital regions. Genetic effects were stronger for cost-efficiency than for other metrics considered, and were more clearly significant in functional networks operating in the $0.09-0.18 \mathrm{~Hz}$ frequency interval than at higher or lower frequencies. These findings are consistent with the hypothesis that brain networks evolved to satisfy competitive selection criteria of maximizing efficiency and minimizing cost, and that optimization of network cost-efficiency represents an important principle for the brain's functional organization.

\section{Introduction}

Functional magnetic resonance imaging (fMRI) studies have shown that regional fluctuations of spontaneous brain activity, measured in the absence of an explicit task (the so-called resting state), are highly organized, being correlated across spatially distributed networks in a manner that recapitulates the topography of task-evoked functional coactivation patterns (Fox and Raichle, 2007; Smith et al., 2009). These networks are robust (Damoiseaux et al., 2006), are linked to synchronized oscillations in neuronal activity (He et al., 2008), and represent a major source of variance in task-evoked activation and behavior (Fox et al., 2007), suggesting that they reflect a functionally important aspect of brain organization (Fox and Raichle, 2007).

\footnotetext{
Received Sept. 16, 2010; revised Nov. 30, 2010; accepted Jan. 2, 2011.

This work was supported by a National Health and Medical Research Council (NHMRC) Project Grant and the lan Potter Foundation. A.F. was supported by an NHMRC O Martin Fellowship (ID: 454797). A.Z. is supported by the Australian Research Council, Discovery Project 0986320. M.Y. and S.J.W. were supported by an NHMRC Clinical Career Development Award. D.S.B. was supported by the National Institutes of Health/Cambridge Graduate Training program. We thank Drs. Sophie Achard and Sarah Medland for helpful advice.

Correspondence should be addressed to Dr. Alex Fornito, Melbourne Neuropsychiatry Centre, Levels 2 and 3, 161 Barry Street, Carlton South, VIC 3053, Australia. E-mail: fornitoa@unimelb.edu.au.

DOI:10.1523/JNEUROSCI.4858-10.2011

Copyright $\odot 2011$ the authors $\quad 0270-6474 / 11 / 313261-10 \$ 15.00 / 0$
}

The nontrivial configuration of spontaneous brain activity suggests that certain principles of network organization may confer adaptive benefits. One proposed principle involves satisfying competitive selection criteria of maximizing communication efficiency and minimizing connection costs (Bullmore and Sporns, 2009). In complex networks such as the brain, communication efficiency is enhanced when the number of connections, or path length, linking any two nodes is low, resulting in faster, more accurate information transmission (Latora and Marchiori, 2001). In anatomical brain networks, a few long-range projections can dramatically increase efficiency (Buzsáki et al., 2004), but this comes at the cost of increased wiring length. In principle, network efficiency could be maximized by adding more connections (Achard and Bullmore, 2007), but the metabolic costs associated with forming and maintaining each brain connection (Laughlin and Sejnowski, 2003) limit the total number sustainable. This balance, between efficiency maximization and connection cost minimization, may be construed as one concerned with optimization of network cost-efficiency.

Cost and efficiency constraints on anatomical brain networks have been studied widely (Cherniak et al., 2004; Chen et al., 2006; 
Kaiser and Hilgetag, 2006; Bassett et al., 2010), but there are reasons to believe that cost-efficiency optimization is also an important principle for functional brain organization. Resting-state functional networks are correlated with underlying anatomical connectivity (Vincent et al., 2007; Honey et al., 2009) and show a small-world organization (Achard et al., 2006) characterized by high efficiency and low cost (Latora and Marchiori, 2001, 2003). Moreover, cost-efficiency may index functional network integrity, being affected by aging and disease and positively correlated with cognitive performance (Achard and Bullmore, 2007; Bassett et al., 2009). Consequently, it has been proposed that both functional and structural brain network organization may represent the outcome of evolutionary selection for neural connections conferring high efficiency at low cost (Kaiser and Hilgetag, 2006; Bullmore and Sporns, 2009; Bassett et al., 2010). This hypothesis predicts that individual differences in functional network cost-efficiency should be under genetic control and therefore demonstrably heritable.

We tested this prediction using resting-state fMRI (rs-fMRI) in healthy monozygotic (MZ) and dizygotic (DZ) twins. Twins provide an ideal natural experiment that enables determination of genetic and environmental contributions to a given phenotype. MZ twins have identical genomes, whereas $\mathrm{DZ}$ twins share, on average, $50 \%$ of their genes. Thus, a phenotype is deemed to be under genetic control, or heritable, if MZ twin covariation exceeds DZ twin covariation.

\section{Materials and Methods}

Participants. Thirty-four (18 male) monozygotic (MZ) and twenty-six (12 male) same-sex dizygotic (DZ) psychiatrically and neurologically healthy twins were recruited for scanning. Twin pairs were registrants with the Australian Twin Registry (ATR), and were recruited through mail-outs and advertisements. All participants were screened using structured clinical interviews (National Institute of Mental Health, 1992). To confirm zygosity, blood samples extracted from the twins were assessed using an AmpFLSTR Profile Plus PCR Amplification Kit (Applied Biosystems). Amplicon allele size ranges were between 100 and 360 bp for each of the following loci: AMEL (X, Y alleles), D13S317 (8 alleles), D18S51 (23 alleles), D21S11 (24 alleles), D3S1358 (8 alleles), D5S818 (10 alleles), D7S820 (10 alleles), D8S1179 (12 alleles), FGA (28 alleles), and vWA (14 alleles). There were no differences between MZ and DZ twins in terms of age $(\mathrm{MZ}$ mean $=37.81$ years, $\mathrm{SD}=13.62$ years; $\mathrm{DZ}$ mean $=$ $43.15, \mathrm{SD}=9.91$ years; $t_{(27)}=-1.16, p=0.26$ ) or intelligence, as estimated using the Wechsler Adult Reading Test (The Psychological Corporation, 2001) $(\mathrm{MZ}$ mean $=107.30, \mathrm{SD}=7.80 ; \mathrm{DZ}$ mean $=106.88$, $\left.\mathrm{SD}=6.07 ; F_{(1,27)}=0.04, p=0.84\right)$. Data from one male MZ twin pair were excluded due to one member moving excessively in the scanner, resulting in $32 \mathrm{MZ}$ twins (16 pairs) and 26 DZ twins (13 pairs) being included in the final analysis. All participants gave written, informed consent before participating, in accordance with local ethics committee guidelines.

Image acquisition and preprocessing. Participants were scanned using a 1.5 T Siemens Avanto located at St Vincent's Hospital, Melbourne, Australia. Eight hundred and forty echo-planar imaging volumes providing $\mathrm{T} 2{ }^{*}$-weighted blood oxygenation level-dependent (BOLD) contrast were acquired with the following parameters: number of slices, 21 ; voxel dimensions, $3.4 \times 3.4 \times 4 \mathrm{~mm}$ ( $1 \mathrm{~mm}$ interslice gap); flip angle, $78^{\circ}$; echo time, $26 \mathrm{~ms}$; time to repetition, $1420 \mathrm{~ms}$. Participants were instructed to lie quietly in the scanner with their eyes closed without falling asleep. All participants confirmed that they did not fall asleep in subsequent debriefing. T1-weighted anatomical scans were also acquired: voxel dimensions $=0.94 \times 0.94 \times 1.5$; flip angle $=15^{\circ}$; echo time $=3.93 \mathrm{~ms}$; time to repetition $=1930 \mathrm{~ms}$.

For each individual, functional volumes were realigned using a rigidbody transformation to correct for geometric displacements associated with head movements and rotations. Temporal motion correction was then performed by regressing the current and lagged first- and secondorder displacements against the time series of the realigned images, as implemented in CamBa (http://www-bmu.psychiatry.cam.ac.uk/ software). The residuals of this regression were used for further analysis. Finally, the realigned, temporally corrected images were spatially normalized to the International Consortium for Brain Mapping echo-planar imaging template supplied with SPM5 (http://www.fil.ion.ucl.ac.uk/ $\mathrm{spm} /$ software/spm5) using a 12-parameter affine transformation (Jenkinson and Smith, 2001) (http://www.fmrib.ox.ac.uk/fsl).

Cortical parcellation. We used a graph analytic approach (Bullmore and Sporns, 2009; Rubinov and Sporns, 2010) to measure the topological properties of each individual's functional brain network. In this framework, the brain is modeled as a graph comprising $N$ nodes connected by $M$ edges. The nodes can be defined at the voxel level (van den Heuvel et al., 2008; Hayasaka and Laurienti, 2010), region level (Achard et al., 2006), or intermediate scales (Hagmann et al., 2007; Fornito et al., 2010; Zalesky et al., 2010). For the current analysis, we parcellated each participant's cortex into 1041 discrete regions using an approach similar to that used by previous authors (Hagmann et al., 2007; Honey et al., 2009). We have previously found that this approximate resolution of analysis provides a good trade-off between computational speed, spatial resolution, and signal-to-noise ratio (Fornito et al., 2010).

To generate the parcellation, each participant's T1-weighted anatomical image was used to generate surface (triangular) mesh models of the gray/white and gray/CSF boundaries of the left and right hemisphere cortical ribbons using validated algorithms (Dale et al., 1999; Fischl et al., 1999) implemented in freely available software (http://surfer.nmr.mgh. harvard.edu/fswiki/Home). The white matter surface of each hemisphere was then parcellated using an algorithm that incrementally merged adjacent pairs of triangles forming the white matter surface mesh. The input to the algorithm was the desired number of regions, denoted with $N$, and the white matter surface triangulation. Let $F$ denote the number of triangles comprising the triangulation. The goal of the algorithm was to achieve a parcellation that maximized the uniformity and compactness of each regions' surface area.

The algorithm comprised two distinct phases. In the first phase, the algorithm was serially performed at a total of $F-N$ merge operations. To complete each merge operation, a pair of adjacent triangles was merged together to form a region. In this sense, a region was simply a set of adjacent triangles. Merge operations were also performed between adjacent pairs of regions, in which case two distinct regions became one. It can be easily shown that performing exactly $F-N$ merge operations ensured that exactly $N$ regions remained at the end of the first phase of the algorithm. In particular, if each triangle is thought of as a distinct region, whenever a pair of regions was merged (regardless of which pair), the total number of regions was reduced by one, and thus after $F-N$ merge operations, $N$ regions must have remained.

While the uniformity in surface area between regions was reasonable, this uniformity was further improved during the second phase of the algorithm, which subdivided any region with a surface area at least twice as large that of the smallest region. This rule guaranteed a strict bound on the uniformity of the final parcellation; namely, the smallest region was guaranteed to be greater than or equal to half the size of the largest region. A result of the second phase of the algorithm was that the final number of regions comprising the parcellation was always slightly greater than the desired number of regions, $N$. In particular, if $M$ regions had a surface area of size at least twice as large as the smallest region, then each of these regions was subdivided into at least two separate regions, and hence the total number of regions comprising the final parcellation was at least $N+$ $M$. Note that a subdivided region was itself subdivided if its surface area was still at least twice as large as the surface area of the smallest region.

In the present study, the algorithm was executed with $N=500$ regions per hemisphere. At the completion of phase 2, the left hemisphere comprised 596 regions, while the right comprised 603. To sample regional activity from the functional volumes, the surface-based parcellations were converted into volumetric representations and projected out to fill the gray matter of the entire cortical ribbon, defined via atlas-based tissue segmentation of the T1-weighted image (Fischl et al., 2002). This volume-based parcellation was dilated by one voxel to ensure adequate coverage of the cortical mantle. Each participant's functional volumes were then registered to their anatomical image via an affine transforma- 


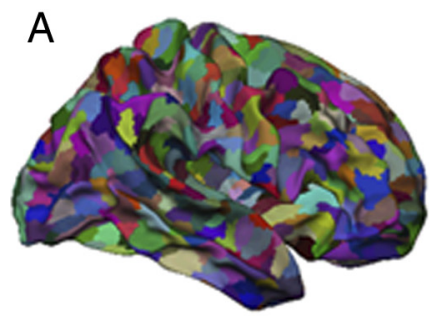

D

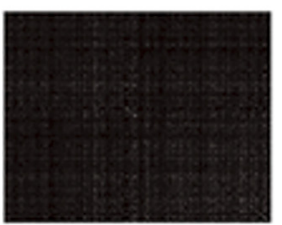

$5 \%$

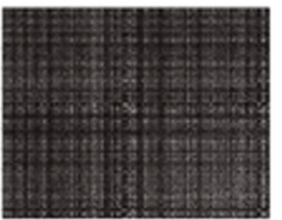

$20 \%$

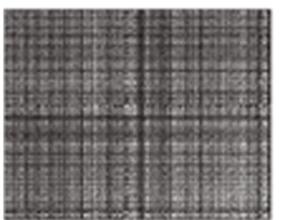

$40 \%$

B

\section{$\rightarrow$}

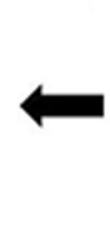

C

Scale 1: $.18-.35 \mathrm{~Hz}$ Scale 2: .09-.18 Hz

Scale 3: $.04-.09 \mathrm{~Hz}$ Scale 4: .02-.04 Hz
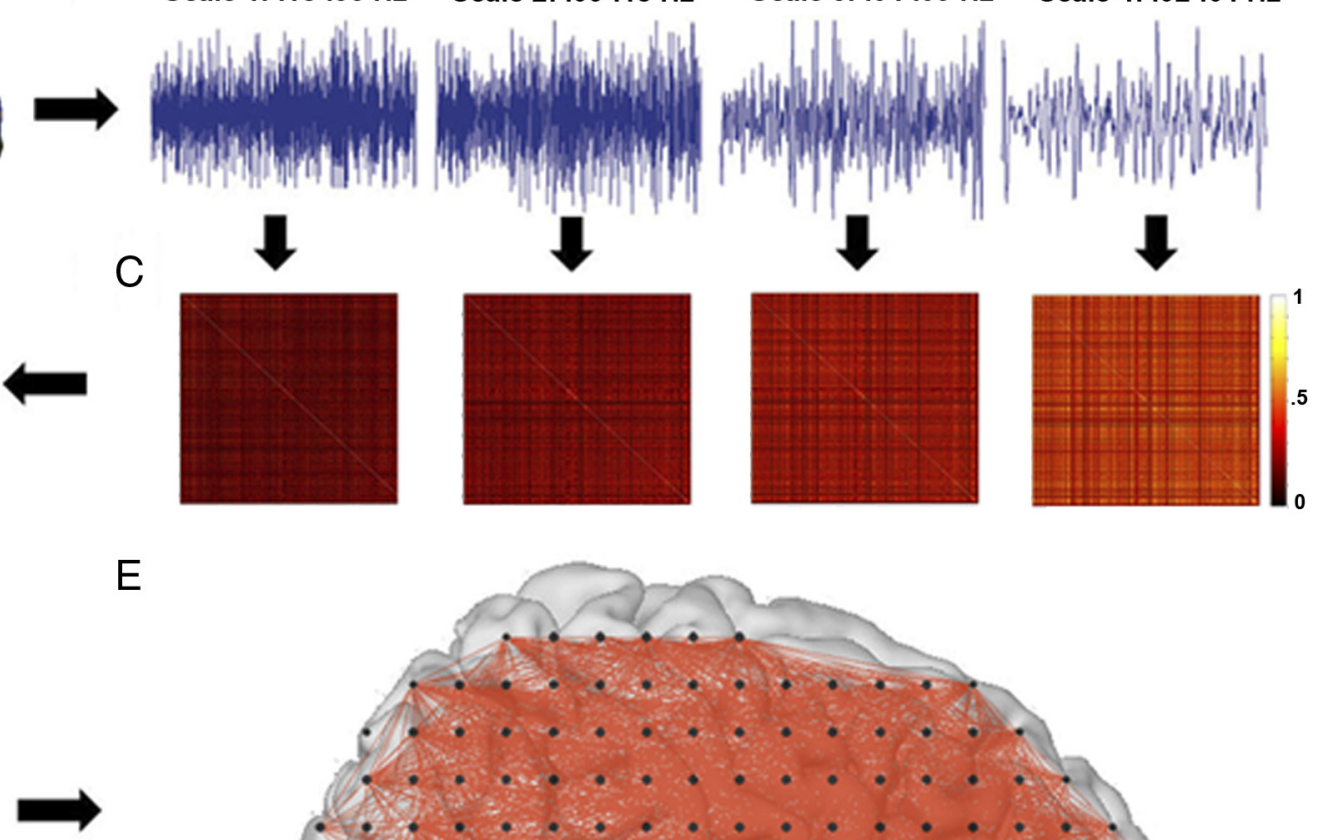

E

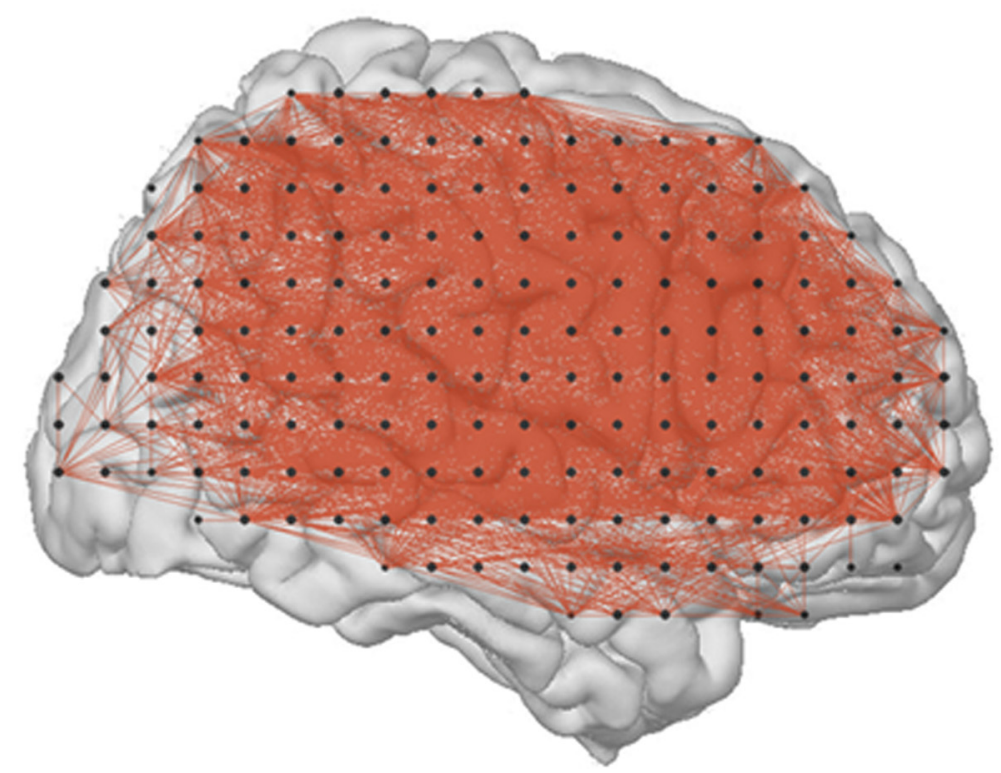

Figure 1. Overview of main processing steps involved in generating graph models of cortical functional connectivity. The cortex of each individual's functional volumes was parcellated using a custom template comprising 1041 regions $(\boldsymbol{A})$. The mean time series of each region was extracted and decomposed into four distinct frequency bands using a wavelet transform $(\boldsymbol{B})$. These filtered time series were corrected for noise, and pairwise correlations between regional wavelet coefficient series were computed to generate a $\{1041 \times 1041\}$ functional connectivity matrix at each wavelet scale (frequency interval) for each participant (C). These functional connectivity matrices were then thresholded at different connection densities (examples of $5 \%$, 20\%, and $40 \%$ are presented) and binarized to generate adjacency matrices $(\boldsymbol{D})$ from which graph models of network topology were constructed $(\boldsymbol{E})$.

tion (Jenkinson and Smith, 2001). To minimize noise introduced by variable EPI coverage across individuals, we excluded ROIs containing $<70 \%$ non-zero intensity voxels, resulting in a final template comprising 1041 cortical regions across both hemispheres (Fig. 1A).

Temporal filtering. The custom template was used to extract the mean time series of each of the 1041 defined cortical regions from each participant's motion-corrected, spatially normalized functional volumes. These mean regional time series were then decomposed into four distinct frequency intervals via the maximal overlap discrete wavelet transform (Percival and Walden, 2000), which is well suited to nonstationary, $1 / f$ type signals such as fMRI measurements (Bullmore et al., 2004). The four frequency intervals (wavelet scales) were as follows: scale 1, 0.18-0.35 $\mathrm{Hz}$; scale 2, 0.09-0.18 Hz; scale 3, 0.04-0.09 Hz; and scale 4, 0.02-0.04 $\mathrm{Hz}$ (Fig. $1 B$ ). We then used the CompCor technique to correct the wavelet-filtered regional time series for fluctuations in non-neuronal physiological processes (e.g., fluctuations in cardiac and respiratory rates) (Behzadi et al., 2007). Briefly, this approach involved the following steps for each participant: (1) identification of voxels showing the highest temporal standard deviation (top 2\%) in the functional data; (2) principal component analysis of these voxels' time courses; (3) identification, via permutation testing, of the components accounting for a significant proportion of variance in the data; (4) extraction of reference time courses for each significant principal component; and (5) correction, via linear regression, of each regional time series for covariance with the principal component time courses identified in step 4 . The results are comparable to those obtained using model-based correction procedures requiring physiological monitoring (Behzadi et al., 2007).

Network characterization. To estimate the cost-efficiency of each individual's cortical functional network, analysis of regional time series progressed according to three broad stages: (1) estimation of interregional functional connectivity; (2) generation of a graph-based representation of network topology; and (3) topological analysis of the network. The details of each of these steps are provided in the following. Detailed accounts of basic principles underlying graph analysis and its application to neuroimaging data have been published previously (Albert and Barabási, 2002; Newman, 2003; Bullmore and Sporns, 2009; Rubinov and Sporns, 2010).

Functional connectivity estimation. To generate cortical functional networks for each participant, correlations between the wavelet-filtered, noise-corrected time series of each possible pair of the 1041 cortical regions sampled were computed separately for each frequency interval (wavelet scale), resulting in four $\{1041 \times 1041\}$ functional connectivity matrices for each individual (Fig. $1 C$ ). A considerable range of correlation values, extending from $<0.01$ to $>0.80$, was observed at all fre- 
quency intervals (supplemental Fig. S1, available at www.jneurosci.org as supplemental material). Average functional connectivity was greater at lower frequencies, consistent with prior observations (Cordes et al., 2001; Achard et al., 2006), but there was also a substantial increase in the estimation error (computed according to Whitcher et al., 2000) associated with lower frequency correlations: from scales 1 to 4 , there was an average $\sim 3$-fold increase in mean estimation error, compared to an $\sim 1$.1-fold increase in average connectivity (supplemental Fig. S1, available at www.jneurosci.org as supplemental material). The increase in estimation error likely reflects the greater statistical difficulties associated with precisely estimating low-frequency properties from relatively short time series (Achard et al., 2008; see also Discussion).

Graph-based representations of brain network topology. The topology of a complex network can be efficiently represented as a graph of $N$ nodes connected by $M$ edges. In the case of brain networks, the nodes represent different brain regions, and the edges represent some measure of interaction between them. In the current analysis, the brain regions represented the 1041 cortical areas from which mean regional fMRI time series were sampled, and the edges represented the frequency-specific wavelet correlations between these time series, computed as described in the previous section.

When networks are generated from continuous association measures, such as wavelet correlations, it is customary to apply a threshold to remove spurious edges and emphasize key topological properties of the network. As this threshold can be arbitrary, we examined network measures across the full range of possible thresholds representing connection densities $5 \%<k<40 \%$; i.e., from $5 \%$ to $40 \%$ of all possible connections (Fig. $1 D$ ). The $5 \%$ lower bound was chosen to avoid excess network fragmentation at lower costs; the $40 \%$ upper bound was chosen because topological properties of brain networks tend toward randomness at higher costs due to the inclusion of potentially spurious functional associations (Bassett et al., 2008).

At each connection density, the graphs were binarized such that suprathreshold correlations were set to 1 and subthreshold correlations to 0 , resulting in a series of unweighted, undirected adjacency matrices representing network connectivity at that threshold [for a similar approach, see Achard et al. (2006) and Achard and Bullmore (2007)]. Graph models were generated from these adjacency matrices by drawing an edge between two nodes if and only if they were linked by a suprathreshold connection (Fig. $1 E$ ).

Topological analysis. Once a graph-based representation has been obtained, numerous measures describing different topological properties of the network can be computed (Bullmore and Sporns, 2009; Rubinov and Sporns, 2010). In this study, we were principally interested in characterizing network cost-efficiency. Four measures were integral to our definition of network cost-efficiency: global communication efficiency $\left(E_{\text {global }}\right)$, regional communication efficiency $\left(E_{\text {regional }}\right)$, connection distance $\left(D_{\text {global }}\right)$, and connection density $(k)$. The connection density, $k$, simply represented the proportion of edges present in the thresholded graph relative to the maximum possible 541,320 connections, i.e., the percentage of suprathreshold edges. The global efficiency of the graph $G$ was calculated following the definition given by Latora and Marchiori (2003):

$$
E_{\text {global }}=\frac{1}{N(N-1)} \sum_{i \neq j \in G} \frac{1}{L_{i j}},
$$

where $N$ is the number of nodes in $G$ and $L_{i j}$ is the shortest path length between nodes $i$ and $j$. Similarly, the efficiency of each node, $i$, was calculated as follows:

$$
E_{\text {regional }}(i)=\frac{1}{(N-1)} \sum_{j \in G} \frac{1}{L_{i j}} .
$$

In general, $E_{\text {global }}$ increases as more connections are added to the network (i.e., as $k$ increases), until it reaches its maximum value when the network is completely connected.

Prior work estimating connection costs in functional networks has simply taken the number of connections present in the network as an index of cost (Achard and Bullmore, 2007; Bassett et al., 2009). However, in physically embedded networks such as the brain, the cost of connecting different nodes scales in proportion to the spatial distance between them (Latora and Marchiori, 2003), and introducing spatial information into the calculation of network costs can alter the results obtained when compared with simple binary measures (Kaiser and Hilgetag, 2006). In this regard, the net connection cost of a network may be viewed as arising from the costs associated with making connections, measured by the connection density of the network, $k$, and the summed physical distance of these connections, which we refer to as the connection distance, $D$. In the absence of a complete map of interregional human brain connectivity that would allow derivation of accurate anatomical estimates of $D$, we followed the approach of Kaiser and Hilgetag (2006) and calculated $D$ as the summed Euclidean distance between regions connected by a suprathreshold connection. Thus, global connection cost was calculated as follows:

$$
D_{\text {global }}=\sum_{i \neq j \in G} d_{i j}
$$

where $d_{i j}=\sqrt{\left(x_{i}-x_{j}\right)^{2}+\left(y_{i}-y_{j}\right)^{2}+\left(z_{i}-z_{j}\right)^{2}}$ and $x, y$, and $z$ define the coordinates of each region's centroid in stereotactic space. Similarly, the regional connection cost was calculated as follows:

$$
D_{\text {regional }}(i)=\sum_{j \in G} d_{i j}
$$

Each of the connection distance measures above was normalized by its corresponding value in a completely connected graph (i.e., when $k=$ $100 \%)$ to scale it between 0 and 1 .

Previous studies taking the difference between efficiency and binary connection cost as an index of network cost-efficiency have found that this difference shows a clear peak at a specific connection density, beyond which the costs associated with adding more connections to the network outweigh the benefits of increased efficiency (Achard and Bullmore, 2007; Bassett et al., 2009). A similar relationship was observed for $E_{\text {global }}$ and $D_{\text {global }}$ in our data. Figure 2, $A$ and $B$, shows how these two measures scale as a function of connection density, $k$, at each wavelet scale. Both measures increase as more connections are added to the network, but the increase in $E_{\text {global }}$ is much steeper at sparser densities $(k<30 \%)$. Due to this discrepancy, taking the difference between $E_{\text {global }}$ and $D_{\text {global }}$ as a simple index of cost-efficiency (Achard and Bullmore, 2007; Bassett et al., $2009)$ reveals a concave curve with a clear peak, $\max \left(E_{\text {global }}-D_{\text {global }}\right)$ (see Fig. $2 C)$. The height of this peak, $\max \left(E_{\text {global }}-D_{\text {global }}\right)$, and the connection density at which it occurred, $k_{\max }$, varied across individuals (see Fig. 2C, inset). Thus, for each individual, the network defined by $k_{\max }$ represented his or her most cost-efficient network configuration: the pivotal connection density at which the cost associated with adding more connections to the network outweighed the benefits of increased efficiency. We therefore defined individual differences in global network cost-efficiency as follows:

$$
\mathrm{CE}_{\text {global }}=\frac{\max \left(E_{\text {global }}-D_{\text {global }}\right)}{k_{\max }} .
$$

Regional cost-efficiency was similarly calculated as follows:

$$
\mathrm{CE}_{\text {regional }}(i)=\frac{E_{\text {regional }}-D_{\text {regional }}}{k_{\max }},
$$

where $E_{\text {regional }}$ and $D_{\text {regional }}$ were calculated from the network defined by each individual's $k_{\max }$.

We normalize by $k_{\max }$ in Equations 5 and 6 to compensate for differences in the connection densities associated with each individual's maximally cost-efficient network, and ensure that when all else was equal, networks with fewer connections were considered to be more costefficient. Thus, by this definition, network connection costs arise from both the distance and number of connections in the network. By exploiting the behavior of the $E_{\text {global }}-D_{\text {global }}$ as a function of $k$, the measure provides a data-driven, individually tailored approach to threshold selec- 
tion because each participant's network is thresholded based on his or her unique maximally cost-efficient network configuration. This approach is therefore more sensitive to individual differences in network organization, which is likely to be an important consideration in twin studies of brain function (Koten et al., 2009). Related measures based on this individually tailored approach have shown stronger correlations with cognitive performance than other network properties (Bassett et al., 2009), supporting the behavioral relevance of our cost-efficiency metric.

We note that the interpretation of connection costs in functional networks can be somewhat ambiguous. In general, the operational costs of a network can be defined in terms of its metabolic requirements, and for a brain network these requirements will scale with the amount of wiring required to connect the network (Laughlin and Sejnowski, 2003). In anatomical networks, connection distance can be clearly related to the wiring cost of the network, since wiring cost scales with the total fiber length used to connect the network. In functional networks, the interpretation is less clear because functional connectivity between two regions may emerge in the absence of a direct anatomical link, presumably through polysynaptic pathways (Vincent et al., 2007; Honey et al., 2009). Nonetheless, it is reasonable to assume that greater wiring and metabolic resources are required to propagate information between two regions that are farther apart than between two spatially adjacent areas (Laughlin and Sejnowski, 2003), whether they are connected directly or through multiple pathways. Thus, connection distance can still be viewed as a proxy estimate of the connection cost of a functional network. Future work integrating fMRI with diffusion-weighted imaging (Skudlarski et al., 2008; Honey et al., 2009; Zalesky and Fornito, 2009) will facilitate more precise characterization of functional connection costs.

In addition to network cost-efficiency, we examined genetic influences on several other commonly studied topological properties of brain networks, assessed across the range of connection densities $5 \%<k<40 \%$, in $5 \%$ increments. These included the component measures making up our cost-efficiency index $-E_{\text {global }}$ and $D_{\text {global }}$, and $E_{\text {global }}-D_{\text {global }}$-in addition to several other network properties commonly studied in the literature: local efficiency $\left(E_{\text {local }}\right)$, normalized path length $(\lambda)$, normalized clustering coefficient $(\gamma)$, and network small-worldness $(\sigma)$. $E_{\text {local }}$ is a measure of local information processing or fault tolerance, and is computed as the efficiency of the subgraph defined by an index node's neighbors after removal of that node (Latora and Marchiori, 2003). To estimate $\lambda$, an index of the global integration of the network, the mean minimum path length between nodes in the observed networks was computed and normalized by the corresponding value computed using a comparable random network. To estimate $\gamma$, the clustering coefficient, a measure of the local cliquishness of network connectivity (i.e., the mean connectedness between each node's neighbors), was calculated in the observed networks and also normalized by its corresponding value in a matched random network. For the normalization, we used the average measure computed from 20 random graphs generated using a rewiring algorithm that ensures matched network size, connection density, and degree distribution (Maslov and Sneppen, 2002). Small-worldness was assessed using the scalar summary $\gamma / \lambda=\sigma$ (Humphries et al., 2006). In small-world networks, $\lambda \approx 1$ and $\gamma>1$, so a nonrandom, small-world topology is apparent when $\sigma>1$. Formal definitions for each of these measures can be found elsewhere (Rubinov and Sporns, 2010).

Structural equation modeling. Similarity between twin pairs (i.e., twin covariance) was assessed using the intraclass correlation coefficient, computed separately for $\mathrm{MZ}\left(r_{\mathrm{MZ}}\right)$ and $\mathrm{DZ}\left(r_{\mathrm{DZ}}\right)$ twins. As a general rule, genetic influences on a given trait are apparent if $r_{\mathrm{MZ}}$ is substantially greater than $r_{\mathrm{DZ}}$. However, we used structural equation modeling to more formally quantify the proportion of variance in each studied phenotype attributable to additive genetic $(A)$, shared environmental $(C)$, and nonshared environmental (E) effects (Neale et al., 2003) (http://www.vcu.edu/mx/). In this context, $A, C$, and $E$ represent latent variables, and their variances $\left(a^{2}, c^{2}\right.$, and $e^{2}$, respectively) reflect the proportion of phenotypic variation attributable to their effects. Model path coefficients were estimated using maximum-likelihood fitting. We first estimated parameters for the full ACE model, and then examined the fit of nested submodels comprising $\mathrm{AE}, \mathrm{CE}$, and $\mathrm{E}$ variance components. Model fit was indexed by -2 times the log-likelihood, which follows a $\chi^{2}$ distribution. However, to avoid inappropriate distributional assumptions on model fits and parametric effects in this relatively modest sample, we used permutation testing for statistical inference (see below). In this context, $p>0.05$ for a given reduced model indicates a fit that is not significantly different from the full ACE model, and should thus be accepted under the rule of parsimony. The significance of A was tested with the $p$ value for the difference in fit between $\mathrm{CE}$ and ACE models; the significance of $\mathrm{C}$ was tested by the $p$ value for the difference in fit between the AE and ACE models (Christian et al., 1995). In both cases, $p<0.05$ indicated a significant effect of that parameter. This modeling procedure assumes that twin covariances are positive, and that $r_{\mathrm{MZ}}>r_{\mathrm{DZ}}$, consistent with a genetic influence on the phenotype under consideration. These assumptions were not met for all the measures studied in our analyses, and so models were not estimated in these cases.

Inferential statistics. At each wavelet scale, or frequency interval, for each phenotypic measure, twin pairings and zygosity (MZ or DZ) labels were permuted 5000 times, with the only constraint being that the original pairings were not broken. Let $T_{i 1}$ and $T_{i 2}$ denote the first and second members of twin pair $i$. If under random reassignment $T_{i 1}$ became paired with $T_{j 1}$, then $T_{i 2}$ would be paired with $T_{j 2}$. Permuting twin pairs in this way ensured exchangeability of observations. At each iteration, the full ACE model, in addition to nested submodels was fitted to the permuted data. The relevant model fit statistics were then extracted to create an empirical null distribution for each parameter. The $p$ value of each effect was given by the percentile of the observed statistic on its corresponding null distribution. Ninety-five percent confidence intervals (CIs) for each parameter were generated using a bootstrapping procedure whereby $\mathrm{MZ}$ and DZ pairs were randomly resampled with replacement to produce a surrogate dataset from which genetic parameters were reestimated. This procedure was iterated 1000 times to yield a sampling distribution for each parameter, from which CIs were calculated according to established procedures (Efron, 1987).

Regional genetic effects were thresholded using a permutation-based, clusterwise procedure. For each of the 1041 regions, we permuted the data 1000 times and reestimated the genetic models, leading to a total of $1.041 \times 10^{6}$ permuted datasets. At each permutation, clusters of regions showing genetic effects were formed by merging adjacent nodes surviving a primary cluster-forming threshold of $p<0.05$. To obtain $p$ values for each cluster that were corrected for multiple comparisons, we followed the procedure advocated by Nichols and Holmes (2002). Specifically, at each permutation, the size of each cluster was computed, and the maximum cluster size obtained at each permutation was retained to form a null distribution against which the observed cluster sizes were evaluated. Evaluating the observed data against the null distribution of the maximal statistic ensured that the resulting $p$ values were corrected for multiple comparisons (Nichols and Holmes, 2002). This method is analogous to the clusterwise correction procedures commonly implemented in traditional voxel-based fMRI studies (Poline et al., 1997; Bullmore et al., 1999). The advantage of cluster-based approaches is that they are more sensitive to spatially extended genetic signals than thresholding based on independent analysis of regional effects.

\section{Results}

\section{Cost-efficiency of human cortical functional networks}

Figure $2 A-C$ illustrates how $E_{\text {global }}, D_{\text {global }}$, and $E_{\text {global }}-D_{\text {global }}$ scaled as a function of connection density, $k$, at each wavelet scale. The positive $E_{\text {global }}-D_{\text {global }}$ values observed at each frequency interval indicate that the functional networks were characterized by high efficiency and relatively low connection cost; i.e., they were cost-efficient. Notably, average $\mathrm{CE}_{\text {global }}$ was higher for wavelet scales 1 and $2(0.18-0.35 \mathrm{~Hz}$ and $0.09-0.18 \mathrm{~Hz}$, respectively) than for lower-frequency scales (Fig. 2D).

\section{Genetic influences on global network cost-efficiency}

To consider genetic effects on global network cost-efficiency, we first examined the MZ and DZ twin correlations for this phenotype at each wavelet scale. For scale $1(0.18-0.35 \mathrm{~Hz})$, twin cor- 
relations were virtually identical $\left(r_{\mathrm{MZ}}=\right.$ 0.44 and $r_{\mathrm{DZ}}=0.45$ ), suggesting minimal genetic influence. In contrast, twin correlations at scale $2(0.09-0.18 \mathrm{~Hz})$ pointed to a pronounced genetic effect, with the $\mathrm{MZ}$ correlation being substantially larger than the DZ correlation $\left(r_{\mathrm{MZ}}=0.61\right.$ and $r_{\mathrm{DZ}}=$ 0.23 ). Structural equation modeling (Neale et al., 2003) was used to partition the phenotypic variance in network cost-efficiency into additive genetic $(A)$, shared environmental $(C)$, and unique environmental/error $(E)$ components, represented by the parameters $a^{2}, c^{2}$, and $e^{2}$, respectively (see Materials and Methods). Additive genetic effects accounted for $60 \%$ of the phenotypic variance in network cost-efficiency at wavelet scale $2\left(a^{2}=0.60,95 \%\right.$ CI: $0.17-$ 0.83 ), with unique environmental effects accounting for the remainder $\left(e^{2}=0.40,95 \%\right.$ CI: $0.19-0.72)$ and shared environmental influences being negligible $\left(c^{2}=0,95 \% \mathrm{CI}\right.$ : $0-0.20)$. Accordingly, a model incorporating only additive genetic and unique environmental components provided the best fit to the data $\left(-2 \mathrm{LL}_{\mathrm{AE}}=97.18, \chi_{(54)}^{2}=0\right.$, $p=1.0 \mathrm{vs}-2 \mathrm{LL}_{\mathrm{CE}}=99.370, \chi_{(54)}^{2}=2.19$, $p=0.02)$.

As with scale 1 , twin correlations at scales $3(0.04-0.09 \mathrm{~Hz})$ and 4 (0.02-0.04 $\mathrm{Hz}$ ) indicated a lack of genetic effects, being very low for both $\mathrm{MZ}$ (scale 3: $r_{\mathrm{MZ}}=$ 0.10; scale 4: $r_{\mathrm{MZ}}=-0.19$ ) and DZ (scale 3: $r_{\mathrm{DZ}}=-0.09$; scale $\left.4: r_{\mathrm{DZ}}=-0.38\right)$ twins. The low and negative twin correlations observed at scales 3 and 4 likely reflect the aforementioned measurement error associated with estimating functional connectivity at these lower frequencies. As a result, genetic parameters were not estimable at these scales (see Discussion).

\section{Genetic influences on regional network cost-efficiency}

We further examined genetic influences on the regional costefficiency, $\mathrm{CE}_{\text {regional }}$, of each cortical node at wavelet scale 2 $(0.09-0.18 \mathrm{~Hz})$. Estimates of $r_{\mathrm{DZ}}$ were relatively low across most cortical regions (median $=0.20$, interquartile range $=0.18$ ), whereas $r_{\mathrm{MZ}}$ values were consistently higher (median $=0.54$, interquartile range $=0.13$ ), particularly in regions of medial and lateral prefrontal cortex, where $r_{\mathrm{MZ}} \geq 0.6$ (Fig. 3).

Genetic models were estimable for $90 \%$ of nodes (see Materials and Methods) and indicated that genetic influences were not distributed homogeneously throughout the cortex: regional heritability $\left(a^{2}\right)$ estimates ranged from $<0.10$ to 0.81 , with a median of 0.51 (Fig. 3). Regions showing statistically significant genetic effects were found throughout the cortex, in a largely bilateral pattern (Fig. 4). These bilateral clusters were found in posterior cingulate, dorsomedial prefrontal cortex (extending onto the superior frontal gyrus), the posterior middle frontal, postcentral, and lingual gyri, the superior parietal lobule, and the ventroposterior bank of the superior temporal sulcus (extending into adjacent temporal and occipital cortices). Additional left-lateralized clusters were found in ventromedial prefrontal, anterior cingulate, primary visual, and parahippocampal cortices; rightlateralized clusters were found in the precuneus, paracentral and $k_{\max }$.
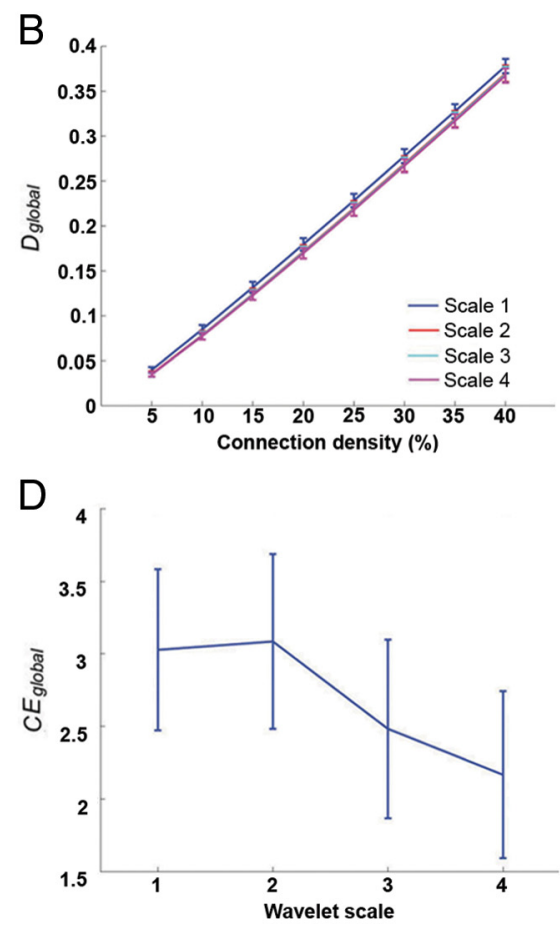

Figure 2. Key global network properties involved in defining network cost-efficiency, as a function of connection density and frequency interval. $\boldsymbol{A}-\boldsymbol{D}$, Sample mean efficiency $\left(E_{\text {global }}\right)(\boldsymbol{A})$; sample mean connection distance $\left(D_{\text {global }}\right)(\boldsymbol{B})$; the difference olobal and $D_{\text {global }}(\boldsymbol{C})$; and global network cost-efficiency $\left(\mathrm{CE}_{\text {global }}\right)$ at each wavelet scale $(\boldsymbol{D})$. Error bars represent $\mathrm{SD}$. Inse

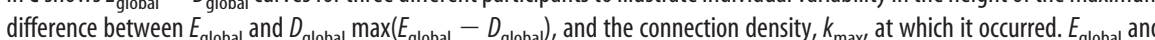
although calculations were based on measurements at $1 \%$ increments to obtain more precise estimates of $\max \left(E_{\text {global }}-D_{\text {global }}\right)$

lobule, and parietal operculum. No statistically significant regional effects of shared environment $\left(c^{2}\right)$ were observed.

\section{Genetic influences on other network properties}

To compare genetic effects on cortical cost-efficiency with other network properties, we also examined twin correlations and genetic parameter estimates for each of the component measures comprising our $\mathrm{CE}_{\text {global }}$ metric (i.e., $E_{\text {global }}, D_{\text {global }}$, and $E_{\text {global }}-$ $D_{\text {global }}$ ) (supplemental Fig. S2, available at www.jneurosci.org as supplemental material), in addition to other commonly studied topological measures: $E_{\text {local }}, \lambda, \gamma$, and $\sigma$ (supplemental Fig. S4, available at www.jneurosci.org as supplemental material). In general, higher-frequency networks (i.e., wavelet scales 1 and 2) showed lower path length and higher clustering, and therefore higher values of the small-worldness parameter, $\sigma$ (supplemental Fig. S3, available at www.jneurosci.org as supplemental material). In particular, networks at wavelet scale $2(0.09-0.18 \mathrm{~Hz})$ were associated with the highest small-worldness values for $k<$ $20 \%$. Networks operating at lower frequencies (wavelet scales 3 and 4) were generally associated with higher local efficiency (supplemental Fig. S3, available at www.jneurosci.org as supplemental material).

Consistent with the cost-efficiency findings, twin correlations were generally low for scales 3 and 4 across all measures, and genetic models were not estimable in most cases. Twin correlations and genetic effects were higher for certain measures in scale 1 and 2 networks, but the results were often dependent on the connection density of the networks. For example, the proportion of variance in $E_{\text {local }}$ attributable to additive genetic effects at scale 
DZ CORRELATION
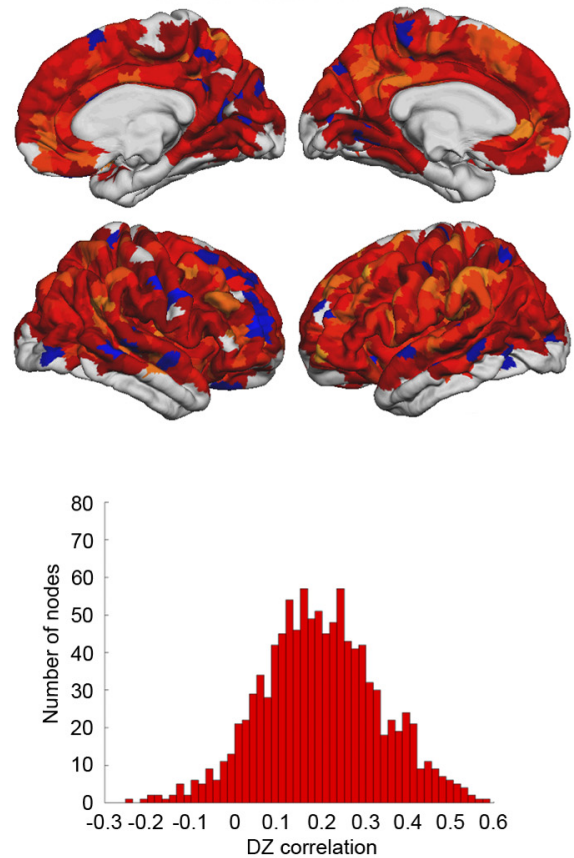

MZ CORRELATION
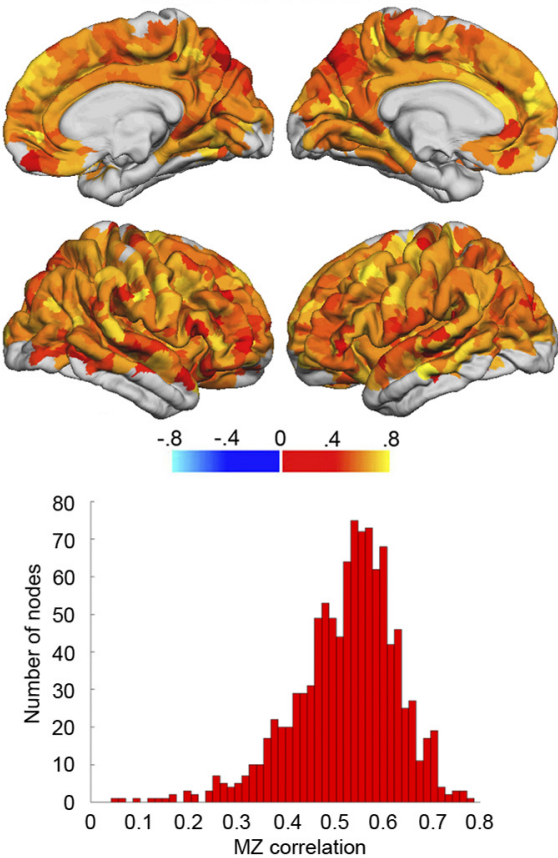
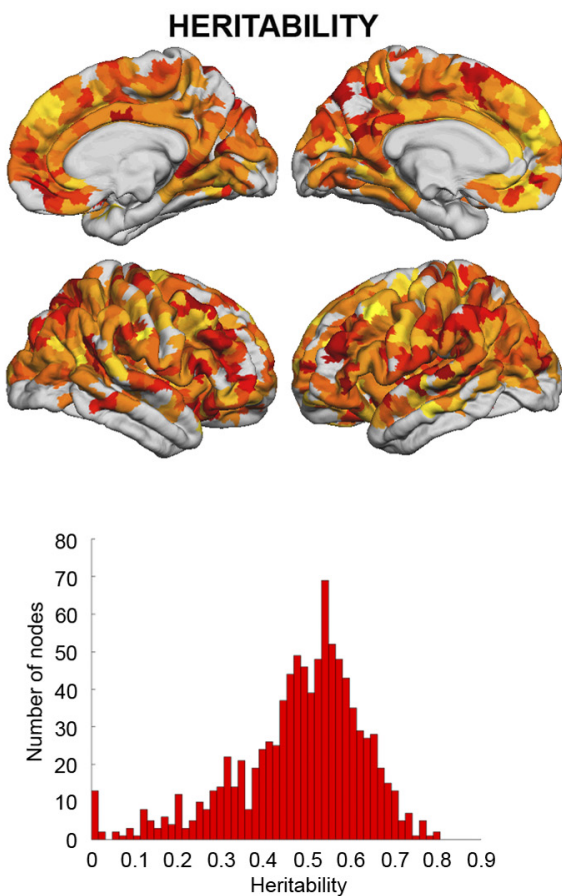

Figure 3. MZ and DZ twin correlations and heritability for regional cost-efficiency of cortical network functional connectivity. Heritability values represent the proportion of variance in regional cost-efficiency attributable to additive genetic factors $\left(a^{2}\right)$. Top, Regional maps of the magnitude of each measure in each region. Bottom, The corresponding frequency histograms of the observed values. For each measure, left hemisphere is presented on the right.

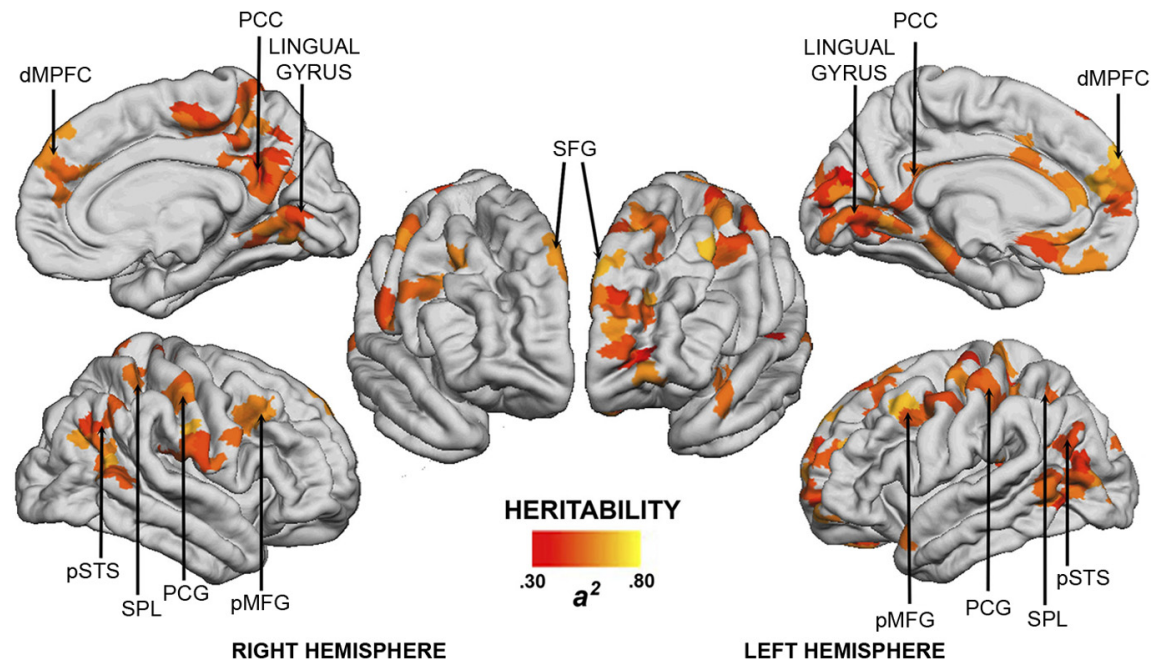

Figure 4. Cortical regions showing statistically significant ( $p<0.05$, corrected) heritability for regional cost-efficiency of network functional connectivity. Arrows highlight regions showing significant genetic effects in both cerebral hemispheres. DMPFC, Dorsomedial prefrontal cortex; $\mathrm{PCC}$, posterior cingulate cortex; $\mathrm{SFG}$, superior frontal gyrus; $\mathrm{PMFG}$, posterior middle frontal gyrus; PCG, postcentral gyrus; SPL, superior parietal lobule; pSTS, posterior superior temporal sulcus. The left hemisphere is presented on the right.

2 showed $>4$-fold variation for different values of $k$ (supplemental Fig. S4, available at www.jneurosci.org as supplemental material). In some cases, heritability estimates were comparable to those obtained for cost-efficiency, though examination of nodal properties suggested the estimates were unreliable. For example, for wavelet scale 2, $a^{2}=0.63$ for $E_{\text {global }}$ at $k=10 \%$ (supplemental Fig. S2, available at www.jneurosci.org as supplemental material), but nodal genetic parameters were estimable for only $10 \%$ of regions due to low and/or negative twin correlations (see Materials and Methods). This suggests that the global effect was an unreliable and unrepresentative index of nodal characteristics. Together, these findings indicate that cost-efficiency of cortical functional connectivity was the most reliably heritable measure of all network organizational properties studied, at both global and regional levels.

\section{Discussion}

The principles underlying cortical network organization have long been debated, largely due to difficulties associated with adequately modeling the brain's largescale connectivity architecture. Our results indicate that one important principle for brain functional networks involves the optimization of connection cost-efficiency. Cost- efficiency of cortical functional connectivity was under strong genetic control, with $60 \%$ of the variance in global network cost-efficiency attributable to genetic factors. In some regions, $>70 \%$ of the variance was accounted for by genetic influences. These heritability estimates are comparable to those reported for whole-brain and regional gray matter volume, which range between 0.66 and 0.97 (Thompson et al., 2001; Peper et al., 2007); cognitive abilities, which range between 0.30 and 0.80 (McClearn et al., 1997; Bouchard, 1998; Boomsma et al., 2002); and personality and psychopathology measures, which range between 0.50 and 0.60 (Boomsma et al., 2002). Thus, cost-efficient organization of cortical functional networks shows heritable influences commensurate with those reported for some of the most widely studied neurobehavioral phenotypes to date. 


\section{The heritability of brain network cost-efficiency}

The benefits of enhancing network communication efficiency are intuitive: lower mean path length between nodes results in faster information transmission and lower probability of signal corruption. Accordingly, increased brain network efficiency has been associated with better cognitive performance (Li et al., 2009; van den Heuvel et al., 2009; Zalesky et al., 2011). Adding more connections will always increase efficiency, but the resulting benefits must be balanced with constraints on the brain's energy expenditure (Laughlin and Sejnowski, 2003). In anatomical brain networks, this constraint has been operationalized as a pressure to minimize wiring costs (Chklovskii et al., 2002; Cherniak et al., 2004; Kaiser and Hilgetag, 2006).

Our findings suggest that cost-efficiency optimization is also an important principle of functional brain organization, consistent with evidence that functional network cost-efficiency predicts cognitive performance and is affected by aging and disease (Achard and Bullmore, 2007; Bassett et al., 2009). There are substantial technical challenges associated with directly measuring metabolic costs in functional networks. The cost measure we used, operationalized in terms of brain network topology, represents a surrogate marker based on the reasonable assumption that the metabolic cost of each connection scales in proportion to its distance (Laughlin and Sejnowski, 2003). However, it remains an indirect cost estimate, and combined analysis of rs-fMRI and diffusion-imaging data (e.g., Honey et al., 2009) should refine this measure in future. Such work could also determine whether genetic effects on functional network cost-efficiency are independent of, or an epiphenomenon of, influences on anatomical network organization, given the correlation between the two (Honey et al., 2009; Zalesky and Fornito, 2009), and the demonstrated genetic effects on white matter architecture (Chiang et al., 2009).

Significant effects were observed throughout the cortex in a largely bilateral pattern. Many of these regions, including left ventromedial prefrontal cortex, right precuneus, and bilateral posterior cingulate, dorsomedial prefrontal and inferior parietal cortices, form part of the so-called default-mode network. This ensemble of regions shows high metabolic activity and functional connectivity when individuals are not engaged in an explicit task (Raichle et al., 2001; Buckner et al., 2008), and its constituent regions act as major connectivity hubs in functional brain networks (Buckner et al., 2009). Additional regions showing significant genetic influences were found in lateral prefrontal and parietal regions, areas known to activate during attentionally demanding tasks (Smith et al., 2009). Many of these regions are implicated as part of a structural core of anatomical brain network connectivity (Hagmann et al., 2008). Thus, one hypothesis is that these network nodes comprise a genetically determined backbone of structural and functional connectivity that facilitates rapid, efficient interregional communication for low connection cost. The widespread, bilateral distribution of these nodes is consistent with their involvement as critical relay points that integrate information from diverse cortical regions.

Glahn et al. (2010) recently reported significant heritability of functional connectivity within the default mode network, and that connectivity within the network was influenced by shared genetic factors. Notably, heritability estimates for their connectivity phenotype were $<0.43$. These are considerably lower than those observed in the current study, and those reported in a recent electroencephalographic study of brain network topology, which were generally $>0.60$ (Smit et al., 2008). This discrepancy may reflect stronger genetic influences on higher-order organizational principles of functional brain networks, such as cost- efficiency optimization. Alternatively, it may reflect sampling and analytic differences, as Glahn et al. (2010) used an extended pedigree design.

Some topological properties related to our cost-efficiency measure also showed high heritability. At some connection densities, up to $63 \%$ of the variance in global efficiency, $E_{\text {global }}$, was attributable to additive genetic effects. This is to be expected, given that our cost-efficiency metric, $\mathrm{CE}_{\text {global }}$, and the measures used to derive it are related by definition. However, heritability estimates in these component measures were less stable for two reasons. First, they were highly contingent on the connection density at which they were studied. An attractive property of our $\mathrm{CE}_{\text {global }}$ measure is that it is computed using individually tailored, data-driven thresholds, which likely increased our sensitivity to detect genetic effects (Koten et al., 2009). Second, nodal genetic parameters for these measures could only be estimated in a minority of cases, as most regions were associated with $r_{\mathrm{MZ}}$ or $r_{\mathrm{DZ}}<$ 0 , or $r_{\mathrm{DZ}}>r_{\mathrm{MZ}}$. Such findings may reflect noisy estimation of twin correlations in a relatively small sample, but the fact that this trend was greatly reversed when examining network costefficiency (e.g., at scale 2, genetic parameters were estimable for $90 \%$ of nodes for $\mathrm{CE}_{\text {global }}$, compared with only $10 \%$ nodes for $E_{\text {global }}$ at $k=10 \%$ ) indicates that our $\mathrm{CE}_{\text {global }}$ measure better captured the genetic variance in network topology. Larger samples may be required to appropriately characterize genetic effects on other measures.

\section{Genetic effects across different frequency intervals}

Twin correlations were low and genetic models unreliable for most network properties studied at wavelet scales 3 and 4, corresponding to the commonly studied $<0.1 \mathrm{~Hz}$ range. One explanation for this finding concerns the error associated with estimating time series correlations, which increases at lower frequencies (Whitcher et al., 2000; Achard et al., 2008) (supplemental Fig. S1, available at www.jneurosci.org as supplemental material). This may be a particularly important consideration when estimating very low-frequency (e.g., $<0.1 \mathrm{~Hz}$ ) properties from limited time series, as acquired during a typical fMRI experiment. Heritability is the proportion of variability in a trait attributable to genetic factors. If measurement error for the trait is large, the proportion of variation attributable to genetic effects will be lower than in a comparable trait measured with less error. This will reduce power for detecting significant genetic influences. In addition, lowerfrequency networks were less cost-efficient (Fig. 2D) and less "small-world," showing both reduced clustering and higher path length (supplemental Fig. S3, available at www.jneurosci.org as supplemental material). These findings are consistent with the lower frequency networks being constructed from noisier functional connectivity estimates. Thus, our findings suggest either that topological properties of low-frequency networks are not significantly heritable or that more reliable estimation of these properties using longer time series may be required to identify any putative genetic influences.

Genetic effects were strongest and most robustly estimated at wavelet scale 2, corresponding to the $0.09-0.18 \mathrm{~Hz}$ interval. Most rs-fMRI experiments filter out signals in this frequency range because signal power is typically highest below $0.1 \mathrm{~Hz}$, and noise from physiological sources can contaminate higher-frequency signal fluctuations (Cordes et al., 2001). While our scale 1 measurements may have been affected by such noise, the scale 2 results are unlikely to be contaminated as the $0.09-0.18 \mathrm{~Hz}$ band is still lower than typical cardiac $(\sim 1 \mathrm{~Hz})$ and respiratory $(\sim 0.3$ $\mathrm{Hz}$ ) cycles (Lowe et al., 1998). 
To control for residual noise, particularly from aliased components of undersampled physiological cycles, our data were corrected using a data-based method shown to be as effective as model-based procedures that incorporate physiological recordings (Behzadi et al., 2007). These considerations, combined with the strong genetic effects observed, suggest that the genetic effects we observed on functional network cost-efficiency do indeed exert their influence on neuronal dynamics.

The functional significance of $0.09-0.18 \mathrm{~Hz}$ BOLD signal oscillations has seldom been investigated, but recent work has found that functional connectivity in this frequency range can distinguish between healthy and patient populations (Garrity et al., 2007; Malinen et al., 2010) and varies in accordance with changing task contexts (Salvador et al., 2008). Collectively, these findings suggest that genetically and behaviorally relevant variance in resting-state BOLD signal fluctuations is manifest at frequencies $>0.10 \mathrm{~Hz}$, consistent with evidence that these fluctuations may possess a broader frequency spectrum than previously thought (for a discussion, see Cole et al., 2010).

\section{Conclusions}

The strong genetic effects observed here support the hypothesis that functional brain networks are organized according to competitive selection criteria of maximizing communication efficiency and minimizing connection costs. An important avenue of future research will involve understanding how such effects relate to other putative organizational principles for the brain (Simon, 1962), and to genetic influences on anatomical connectivity.

\section{References}

Achard S, Bullmore E (2007) Efficiency and cost of economical brain functional networks. PLoS Comput Biol 3:e17.

Achard S, Salvador R, Whitcher B, Suckling J, Bullmore E (2006) A resilient, low-frequency, small-world human brain functional network with highly connected association cortical hubs. J Neurosci 26:63-72.

Achard S, Bassett DS, Meyer-Lindenberg A, Bullmore E (2008) Fractal connectivity of long-memory networks. Phys Rev E Stat Nonlin Soft Matter Phys 77:036104.

Albert R, Barabási AL (2002) Statistical mechanics of complex networks. Rev Mod Phys 74:47-97.

Bassett DS, Bullmore E, Verchinski BA, Mattay VS, Weinberger DR, MeyerLindenberg A (2008) Hierarchical organization of human cortical networks in health and schizophrenia. J Neurosci 28:9239-9248.

Bassett DS, Bullmore ET, Meyer-Lindenberg A, Apud JA, Weinberger DR, Coppola R (2009) Cognitive fitness of cost-efficient brain functional networks. Proc Natl Acad Sci U S A 106:11747-11752.

Bassett DS, Greenfield DL, Meyer-Lindenberg A, Weinberger DR, Moore SW, Bullmore ET (2010) Efficient physical embedding of topologically complex information processing networks in brains and computer circuits. PLoS Comput Biol 6:e1000748.

Behzadi Y, Restom K, Liau J, Liu TT (2007) A component based noise correction method (CompCor) for BOLD and perfusion based fMRI. Neuroimage 37:90-101.

Boomsma D, Busjahn A, Peltonen L (2002) Classical twin studies and beyond. Nat Rev Genet 3:872-882.

Bouchard TJ Jr (1998) Genetic and environmental influences on adult intelligence and special mental abilities. Hum Biol 70:257-279.

Buckner RL, Andrews-Hanna JR, Schacter DL (2008) The brain's default network: anatomy, function, and relevance to disease. Ann N Y Acad Sci 1124:1-38.

Buckner RL, Sepulcre J, Talukdar T, Krienen FM, Liu H, Hedden T, AndrewsHanna JR, Sperling RA, Johnson KA (2009) Cortical hubs revealed by intrinsic functional connectivity: mapping, assessment of stability, and relation to Alzheimer's disease. J Neurosci 29:1860-1873.

Bullmore E, Sporns O (2009) Complex brain networks: graph theoretical analysis of structural and functional systems. Nat Rev Neurosci 10:186-198.

Bullmore ET, Suckling J, Overmeyer S, Rabe-Hesketh S, Taylor E, Brammer
MJ (1999) Global, voxel, and cluster tests, by theory and permutation, for a difference between two groups of structural MR images of the brain. IEEE Trans Med Imaging 18:32-42.

Bullmore E, Fadili J, Maxim V, Sendur L, Whitcher B, Suckling J, Brammer M, Breakspear M (2004) Wavelets and functional magnetic resonance imaging of the human brain. Neuroimage 23 [Suppl 1]:S234-S249.

Buzsáki G, Geisler C, Henze DA, Wang XJ (2004) Interneuron diversity series: circuit complexity and axon wiring economy of cortical interneurons. Trends in neurosciences 27:186-193.

Chen BL, Hall DH, Chklovskii DB (2006) Wiring optimization can relate neuronal structure and function. Proc Natl Acad Sci U S A 103:47234728 .

Cherniak C, Mokhtarzada Z, Rodriguez-Esteban R, Changizi K (2004) Global optimization of cerebral cortex layout. Proc Natl Acad Sci U S A 101:1081-1086.

Chiang MC, Barysheva M, Shattuck DW, Lee AD, Madsen SK, Avedissian C, Klunder AD, Toga AW, McMahon KL, de Zubicaray GI, Wright MJ, Srivastava A, Balov N, Thompson PM (2009) Genetics of brain fiber architecture and intellectual performance. J Neurosci 29:2212-2224.

Chklovskii DB, Schikorski T, Stevens CF (2002) Wiring optimization in cortical circuits. Neuron 34:341-347.

Christian JC, Norton JA Jr, Sorbel J, Williams CJ (1995) Comparison of analysis of variance and maximum likelihood based path analysis of twin data: partitioning genetic and environmental sources of covariance. Genet Epidemiol 12:27-35.

Cole DM, Smith SM, Beckmann CF (2010) Advances and pitfalls in the analysis and interpretation of resting-state FMRI data. Front Syst Neurosci 4:8.

Cordes D, Haughton VM, Arfanakis K, Carew JD, Turski PA, Moritz CH, Quigley MA, Meyerand ME (2001) Frequencies contributing to functional connectivity in the cerebral cortex in "resting-state" data. AJNR Am J Neuroradiol 22:1326-1333.

Dale AM, Fischl B, Sereno MI (1999) Cortical surface-based analysis. I. Segmentation and surface reconstruction. Neuroimage 9:179-194.

Damoiseaux JS, Rombouts SA, Barkhof F, Scheltens P, Stam CJ, Smith SM, Beckmann CF (2006) Consistent resting-state networks across healthy subjects. Proc Natl Acad Sci U S A 103:13848-13853.

Efron B (1987) Better bootstrap confidence intervals. J Am Stat Assoc 82:171-185

Fischl B, Sereno MI, Dale AM (1999) Cortical surface-based analysis. II: inflation, flattening, and a surface-based coordinate system. Neuroimage 9:195-207.

Fischl B, Salat DH, Busa E, Albert M, Dieterich M, Haselgrove C, van der Kouwe A, Killiany R, Kennedy D, Klaveness S, Montillo A, Makris N, Rosen B, Dale AM (2002) Whole brain segmentation: automated labeling of neuroanatomical structures in the human brain. Neuron 33:341-355.

Fornito A, Zalesky A, Bullmore ET (2010) Network scaling effects in graph analytic studies of human resting-state FMRI data. Front Syst Neurosci 4:22.

Fox MD, Raichle ME (2007) Spontaneous fluctuations in brain activity observed with functional magnetic resonance imaging. Nat Rev Neurosci 8:700-711.

Fox MD, Snyder AZ, Vincent JL, Raichle ME (2007) Intrinsic fluctuations within cortical systems account for intertrial variability in human behavior. Neuron 56:171-184.

Garrity AG, Pearlson GD, McKiernan K, Lloyd D, Kiehl KA, Calhoun VD (2007) Aberrant "default mode" functional connectivity in schizophrenia. Am J Psychiatry 164:450-457.

Glahn DC, Winkler AM, Kochunov P, Almasy L, Duggirala R, Carless MA, Curran JC, Olvera RL, Laird AR, Smith SM, Beckmann CF, Fox PT, Blangero J (2010) Genetic control over the resting brain. Proc Natl Acad Sci U S A 107:1223-1228.

Hagmann P, Kurant M, Gigandet X, Thiran P, Wedeen VJ, Meuli R, Thiran JP (2007) Mapping human whole-brain structural networks with diffusion MRI. PLoS ONE 2:e597.

Hagmann P, Cammoun L, Gigandet X, Meuli R, Honey CJ, Wedeen VJ, Sporns O (2008) Mapping the structural core of human cerebral cortex. PLoS Biol 6:e159.

Hayasaka S, Laurienti PJ (2010) Comparison of characteristics between region-and voxel-based network analyses in resting-state fMRI data. Neuroimage 50:499-508. 
He BJ, Snyder AZ, Zempel JM, Smyth MD, Raichle ME (2008) Electrophysiological correlates of the brain's intrinsic large-scale functional architecture. Proc Natl Acad Sci U S A 105:16039-16044.

Honey CJ, Sporns O, Cammoun L, Gigandet X, Thiran JP, Meuli R, Hagmann P (2009) Predicting human resting-state functional connectivity from structural connectivity. Proc Natl Acad Sci U S A 106:2035-2040.

Humphries MD, Gurney K, Prescott TJ (2006) The brainstem reticular formation is a small-world, not scale-free, network. Proc Biol Sci 273:503-511.

Jenkinson M, Smith S (2001) A global optimisation method for robust affine registration of brain images. Med Image Anal 5:143-156.

Kaiser M, Hilgetag CC (2006) Nonoptimal component placement, but short processing paths, due to long-distance projections in neural systems. PLoS Comput Biol 2:e95.

Koten JW Jr, Wood G, Hagoort P, Goebel R, Propping P, Willmes K, Boomsma DI (2009) Genetic contribution to variation in cognitive function: an FMRI study in twins. Science 323:1737-1740.

Latora V, Marchiori M (2001) Efficient behavior of small-world networks. Phys Rev Lett 87:198701.

Latora V, Marchiori M (2003) Economic small-world behavior in weighted networks. Eur Phys J B 32:249-263.

Laughlin SB, Sejnowski TJ (2003) Communication in neuronal networks. Science 301:1870-1874

Li Y, Liu Y, Li J, Qin W, Li K, Yu C, Jiang T (2009) Brain anatomical network and intelligence. PLoS Comput Biol 5:e1000395.

Lowe MJ, Mock BJ, Sorenson JA (1998) Functional connectivity in single and multislice echoplanar imaging using resting-state fluctuations. Neuroimage 7:119-132.

Malinen S, Vartiainen N, Hlushchuk Y, Koskinen M, Ramkumar P, Forss N, Kalso E, Hari R (2010) Aberrant temporal and spatial brain activity during rest in patients with chronic pain. Proc Natl Acad Sci U S A 107:6493-6497.

Maslov S, Sneppen K (2002) Specificity and stability in topology of protein networks. Science 296:910-913.

McClearn GE, Johansson B, Berg S, Pedersen NL, Ahern F, Petrill SA, Plomin R (1997) Substantial genetic influence on cognitive abilities in twins 80 or more years old. Science 276:1560-1563.

Neale MC, Boker SM, Xie G, Maes HH (2003) Mx: statistical modeling. Box 980126 MCV, Richmond, VA 23298.

Newman MJE (2003) The structure and function of complex networks. SIAM Rev 45:167-256.

Nichols TE, Holmes AP (2002) Nonparametric permutation tests for functional neuroimaging: a primer with examples. Hum Brain Mapp 15:1-25.

National Institute of Mental Health (1992) Family interview for genetic studies. Bethesda, MD: National Institute of Mental Health.

Peper JS, Brouwer RM, Boomsma DI, Kahn RS, Hulshoff Pol HE (2007) Genetic influences on human brain structure: a review of brain imaging studies in twins. Hum Brain Mapp 28:464-473.

Percival DB, Walden AT (2000) Wavelet methods for time series analysis. Cambridge, UK: Cambridge UP.

Poline JB, Worsley KJ, Evans AC, Friston KJ (1997) Combining spatial ex- tent and peak intensity to test for activations in functional imaging. Neuroimage 5:83-96.

Raichle ME, MacLeod AM, Snyder AZ, Powers WJ, Gusnard DA, Shulman GL (2001) A default mode of brain function. Proc Natl Acad Sci U S A 98:676-682.

Rubinov M, Sporns O (2010) Complex network measures of brain connectivity: uses and interpretations. Neuroimage 52:1059-1069.

Salvador R, Martínez A, Pomarol-Clotet E, Gomar J, Vila F, Sarró S, Capdevila A, Bullmore E (2008) A simple view of the brain through a frequencyspecific functional connectivity measure. Neuroimage 39:279-289.

Simon HA (1962) The architecture of complexity. Proc Am Philos Soc 106:467-482.

Skudlarski P, Jagannathan K, Calhoun VD, Hampson M, Skudlarska BA, Pearlson G (2008) Measuring brain connectivity: diffusion tensor imaging validates resting state temporal correlations. Neuroimage 43:554561.

Smit DJ, Stam CJ, Posthuma D, Boomsma DI, de Geus EJ (2008) Heritability of "small-world" networks in the brain: a graph theoretical analysis of resting-state EEG functional connectivity. Hum Brain Mapp 29:1368-1378.

Smith SM, Fox PT, Miller KL, Glahn DC, Fox PM, Mackay CE, Filippini N, Watkins KE, Toro R, Laird AR, Beckmann CF (2009) Correspondence of the brain's functional architecture during activation and rest. Proc Natl Acad Sci U S A 106:13040-13045.

The Psychological Corporation (2001) Wechsler test of adult reading: manual. San Antonio: The Psychological Corporation.

Thompson PM, Cannon TD, Narr KL, van Erp T, Poutanen V-P, Huttunen M, Lönnqvist J, Standertskjöld-Nordenstam CG, Kaprio J, Khaledy M, Dail R, Zoumalan CI, Toga AW (2001) Genetic influences on brain structure. Nat Neurosci 4:1253-1258.

van den Heuvel MP, Stam CJ, Boersma M, Hulshoff Pol HE (2008) Smallworld and scale-free organization of voxel-based resting-state functional connectivity in the human brain. Neuroimage 43:528-539.

van den Heuvel MP, Stam CJ, Kahn RS, Hulshoff Pol HE (2009) Efficiency of functional brain networks and intellectual performance. J Neurosci 29:7619-7624.

Vincent JL, Patel GH, Fox MD, Snyder AZ, Baker JT, Van Essen DC, Zempel JM, Snyder LH, Corbetta M, Raichle ME (2007) Intrinsic functional architecture in the anaesthetized monkey brain. Nature 447:83-86.

Whitcher B, Guttorp P, Percival DB (2000) Wavelet analysis of covariance with application to atmospheric time series. J Geophys Res Atmos 105:14941-14962.

Zalesky A, Fornito A (2009) A DTI-derived measure of cortico-cortical connectivity. IEEE Trans Med Imaging 28:1023-1036.

Zalesky A, Fornito A, Harding IH, Cocchi L, Yücel M, Pantelis C, Bullmore ET (2010) Whole-brain anatomical networks: does the choice of nodes matter? Neuroimage 50:970-983.

Zalesky A, Fornito A, Seal ML, Cocchi L, Westin CF, Bullmore ET, Egan GF, Pantelis C (2011) Disrupted axonal fiber connectivity in schizophrenia. Biol Psychiatry 69:80-89. 